\title{
CURRENT LITERATURE
}

\section{Epileptogenesis and Neurogenesis Share the Same Genes}

\author{
Overlapping Microarray Profiles of Dentate Gyrus \\ Gene Expression during Development- and \\ Epilepsy-associated Neurogenesis and Axon \\ Outgrowth
}

Elliott RC, Miles MF, Lowenstein DH

J Neurosci 2003;23(6):2218-2227

Neurogenesis and axon outgrowth are features shared by normal nervous system development and certain forms of epileptogenesis. This observation led to the hypothesis that some aspects of normal development and epileptogenesis have common molecular mechanisms. To test this hypothesis, we used DNA microarray analysis to characterize gene expression in the dentate gyrus and to identify genes exhibiting similar patterns of regulation during development and epileptogenesis. Of more than 8,000 sequences surveyed, more than 600 were regulated during development or epileptogenesis, and 37 of these were either upregulated or downregulated during both processes. In situ hybridization analysis of a subset of these "commonality genes" confirmed the patterns of regulation predicted by the microarray data in most cases and demonstrated various spatial and temporal patterns of commonality gene expression. Of the 25 named commonality genes in which some functional characteristics are known, 11 have been implicated in cell morphology and axon outgrowth or cellular proliferation and fate determination. This enrichment for candidate plasticity-related genes supports the concept that developmental mechanisms contribute to network alterations associated with epileptogenesis and offers a useful strategy for identifying molecules that may play a role in both of these processes.

\section{COMMENTARY}

E lliott et al. posit that the molecules guiding dentate granule cell neurogenesis and axon outgrowth during nervous system development overlap with those expressed during epileptogenesis. This is a hypothesis for which support already exists from numerous studies conducted over the last decade, show- ing that developmentally regulated molecules are reexpressed in the dentate gyrus after status epilepticus. However, the present study differs because it uses advanced microarray analyses to identify these "commonality genes," which are expressed in development (i.e., postnatal day 3 ) and 14 days after pilocarpineinduced status epilepticus in adult rats.

Changes in dentate gyrus granule cell gene expression, which transpire during both development and epileptogenesis, were broadly evaluated by using oligonucleotide-based DNA microarrays-representing close to 7,000 named genes and more than 1,000 expressed sequence tags (ESTs). The arrays were used to probe labeled complementary RNA (cRNA) derived from microdissected dentate gyrus tissue samples of experimental and control animals. In the developmental analysis, microarray data from postnatal day 3 rats were compared with those from naïve adult animals. In the epileptogenesis analysis, microarray data from 14-day, post-status epilepticus rats were compared with those from saline-treated adult animals. After removal of hypervariable expression between control samples, the data were subjected to specific statistical criteria that the authors themselves derived.

The genes that were significantly upregulated or downregulated during development or epileptogenesis were identified and then tabulated. As expected, a greater degree of transcriptional regulation occurs during development than during epileptogenesis, with 509 genes/ESTs being up- or downregulated in development, compared with 129 being regulated during epileptogenesis. Overlap of gene expression in the two processes also was demonstrated, although the overlap was skewed significantly toward downregulated genes. Genes regulated in development or epileptogenesis included immediate-early response genes, calcium homeostasis genes, cell-signaling genes, cell-cycle and fate-determination genes, morphology and structure genes, and injury/survival genes. Only 14 genes/ESTs were upregulated in both development and epileptogenesis, and these were mainly morphology genes and cell-cycle/fate genes, with a few metabolic and injury/survival genes. A total of 23 commonality genes were downregulated and are involved mainly in calcium homeostasis, metabolism, morphology, and extracellular signaling.

This study was a heroic effort, involving a vast array of statistical analyses at various steps in addition to the laborious molecular biology. The chip used represents 8,000 sequences, amounting to $25 \%$ of the rat genome. The statistical analyses 
performed are essentially designed to separate signal from noise. Calculations of average difference measures among gene expression, normalization, and scaling factors used were performedeach implemented to separate true trends in the data (signal) from spurious, highly variable genes and from other false effects. To aid their research, Elliott et al. developed a novel algorithm, termed an "S-score" analysis, designed to determine the likelihood that the hybridization signal for a given gene was different between two arrays. However, the researchers note that because of difficulties in testing statistical rigor of their algorithm, the gene commonality demonstrated in their analysis can be regarded as representing only trends. The lack of specificity is attributable to the derivation, and hence, to the lack of independence of the commonality gene group from the groups of development- and epileptogenesis-regulated genes, as well as to the lack of complete, uniform annotation of each of the named genes that was used on the Affymetrix microarray.

In an effort to check their findings independently, the authors searched the literature for corroboration of the 25 named commonality genes. Such data come largely from studies in which single genes were explored. Roughly half of the named commonality genes have previously been shown to be developmentally regulated in a manner consistent with the array data. Among these genes, three were reported to be regulated in hippocampus after seizures or status epilepticus. Some of the commonality genes, indeed, demonstrate quite compelling associations with development and seizures (for example, neuropeptide $Y$, the endogenous regulator of neuronal excitability believed to have anticonvulsant properties; thymosin $\beta-10$, the actin-sequestering peptide involved in morphology; and Sox11, the transcription factor implicated in cell-cycle/fate determination, all are upregulated commonality genes). Similarly, the commonality genes coding for the neuronal calcium sensor proteins, NVP-2 and hippocalcin, and the calcium-binding protein calbindin were all downregulated.

Nonetheless, several important issues were not addressed by this study. For instance, regarding the 37 commonality genes identified between development and status epilepticus, no context is provided to judge this number as "high" or "low." The authors also do not address how their findings should be assessed in relation to the $75 \%$ of the rat genome that was neglected (i.e., not present on the microarray chip) in their study. Likewise, no comment was made about potential confounding factors of the microdissection process itself, which requires 20 minutes to complete. It is plausible that up- or downregulation of genes could occur over this time, and the reader is not informed as to whether the cold temperatures used were sufficient to stop ongoing molecular processing. The procedure for obtaining tissue (even for control tissue) might trigger alterations in gene production, providing spurious overlapping genes. What were the controls used for microdissection process? Correspondingly, no procedural information is provided for the anesthesia and injections given to the rodents. Moreover, it is unclear that the animal groups that were compared would experience euthanasia in the same way, allowing differential molecular, and perhaps genomic processes, to ensue before or after death in postnatal day 3 rats as compared with naïve adult rats and resulting in gene products for death rather than the experimental target. Finally, with such a sensitive and potent technique, the possibility of iatrogenic influences should always be entertained. Presumably, all these issues were addressed, but inclusion of such information is appropriate.

Nevertheless, the technique used is a powerful one, designed to screen thousands of genes, including several genes not yet even named and many genes for which no product is known, and identify specific genes that are up- or downregulated. The technique is of immense heuristic value; however, because of its heavy reliance on statistical methods, it lacks quantitative rigor. The research clearly demonstrated that the use of microarray technology is capable of confirming prior findings, but because of the vagaries of the technique, any novel outcomes will have to await corroboration by other means before gaining general acceptance.

by Larry S. Benardo, M.D., Ph.D. 